\title{
Breakup of shells under explosion and impact
}

\author{
F. K. Wittel, ${ }^{1}$ F. Kun, ${ }^{2, *}$ H. J. Herrmann, ${ }^{3}$ and B. H. Kröplin ${ }^{1}$ \\ ${ }^{1}$ Institute for Statics and Dynamics of Aerospace Structures, University of Stuttgart, Pfaffenwaldring 27, 70569 Stuttgart, Germany \\ ${ }^{2}$ Department of Theoretical Physics, University of Debrecen, P. O. Box 5, H-4010 Debrecen, Hungary \\ ${ }^{3}$ ICP, University of Stuttgart, Pfaffenwaldring 27, D-70569 Stuttgart, Germany \\ (Received 6 July 2004; revised manuscript received 22 October 2004; published 10 January 2005)
}

\begin{abstract}
A theoretical and experimental study of the fragmentation of closed thin shells made of a disordered brittle material is presented. Experiments were performed on eggshells under two different loading conditions: fragmentation due to an impact with a hard wall and explosion by a combustion mixture giving rise to power law fragment size distributions. For the theoretical investigations a three-dimensional discrete element model of shells is constructed. Molecular dynamics simulations of the two loading cases resulted in power law fragment mass distributions in satisfactory agreement with experiments. Based on large scale simulations we give evidence that power law distributions arise due to an underlying phase transition which proved to be abrupt and continuous for explosion and impact, respectively. Our results demonstrate that the fragmentation of closed shells defines a universality class, different from that of two- and three-dimensional bulk systems.
\end{abstract}

DOI: 10.1103/PhysRevE.71.016108

PACS number(s): 64.60.Ak, 46.50.+a

\section{INTRODUCTION}

Closed shells made of solid materials are often used in everyday life, industrial applications, and engineering practice as containers, pressure vessels, or combustion chambers. From a structural point of view, aircraft vehicles, launch vehicles like rockets, and building blocks of a space station are also shell-like systems, and even certain types of modern buildings can be considered as shells. The eggshell as nature's oldest container proved to be a reliable construction for protecting life. In most of the applications shell-like constructions operate under an internal pressure much higher than the surrounding one. Hence, careful design and optimization of structural and material properties is required to ensure the stability and reliability of the system. Closed shells usually fail due to an excess internal load which can arise either as a result of slowly driving the system above its stability limit during its usage or service time, or by a pressure pulse caused by an explosive shock inside the shell. Due to the widespread applications, the failure of shell systems is a very important scientific and technological problem which also has an enormous social impact due to the human costs arising, for instance, in accidental events.

Fragmentation, i.e. the breaking of particulate materials into smaller pieces, is abundant in nature and underlies several industrial processes, which have attracted continuous interest in scientific and engineering research over recent decades [1-17]. Fragmentation phenomena can be observed on a broad range of length scales ranging from the collisional evolution of asteroids and meteor impacts on the astrophysical scale, through geological phenomena and industrial applications on the intermediate scale, down to the breakup of large molecules and heavy nuclei on the atomic scale. In laboratory experiments on the fragmentation of solids, the energy input is usually achieved by shooting a projectile into

\footnotetext{
*Electronic address: feri@dtp.atomki.hu
}

a solid block [4-9], making an explosion inside the sample $[2,3]$, or the collision of macroscopic bodies (free fall impact) [11-17]. Due to the violent nature of the process, observations on fragmenting systems are often restricted to the final state, making the fragment size (volume, mass, charge, etc.) the main characteristic quantity. The most striking observation on fragmentation is that the distribution of fragment sizes shows a power law behavior, independent of the way of imparting energy, relevant microscopic interactions, and length scales involved, with an exponent depending only on the dimensionality of the system [2-18]. During recent years experimental [2-18] and theoretical [19-42] efforts focused on the validity region and the reason for the observed universality in one, two, and three dimensions. Detailed studies have revealed that universality prevails for large enough input energies when the system falls apart into small enough pieces [7-10,13-17,19-28]; however, at lower energies a systematic dependence of the exponent on the input energy was evidenced $[29,30]$. Recent investigations on the low energy limit of fragmentation suggest that the power law distribution of fragment sizes arises due to an underlying critical point $[22,23,28,35,36,39]$.

In addition to the industrial and social impact of the failure of shell-like systems, they are also of high scientific importance for the understanding of fragmentation phenomena. Former studies on fragmentation have focused on the behavior of bulk systems in one, two, and three dimensions under impact and explosive loading; however, hardly any studies have been devoted to fragmentation of shells [39]. The peculiarity of the breakup of closed shells originates from the fact that the local structure is inherently two dimensional; however, the dynamics of the system, the motion of material elements, deformation, and stress states are three dimensional, which allows for a rich variety of failure modes [39].

In this paper we present a detailed experimental and theoretical study of the fragmentation of closed solid shells arising due to an excess load inside the shell. Experiments were performed on brown and white hen eggshells and on quail eggshells under two different loading conditions: fragmenta- 
tion due to an impact with a hard wall and explosion by a combustion mixture have been considered, resulting in power law fragment size distributions. For simplicity, our theoretical study is restricted to spherical shells such that a three-dimensional discrete element model of spherical shell systems was worked out. In molecular dynamics simulations of the two loading cases, power law fragment mass distributions were obtained in satisfactory agreement with experiments. Based on large scale simulations we give evidence that power law distributions arise due to an underlying phase transition which proved to be abrupt for explosion and continuous for impact. Analyzing the energetics of the explosion process in the two loading cases and the evolution of the fragment mass distributions, we demonstrate that the fragmentation of closed shells defines a universality class different from that of two- and three-dimensional bulk systems.

\section{EXPERIMENTS}

Hen eggs provide an excellent possibility for the study of fragmentation of thin brittle shells of disordered materials with the additional advantages of being cheap and easy to handle, making the patience of scientists the only limiting factor for the subsequent improvement of the experimental results. Our experiments were performed on ordinary brown hen eggshells. In the preparation, first two holes of regular circular shape were drilled on the bottom and top of the egg through which the content of the egg was blown out. The inside was carefully washed and rinsed out several times and finally the empty shells were dried in a microwave oven to get rid of all moisture of the eggshell. The drying process proved to be essential to ensure that the cuticula, which cannot be blown out, completely loses its toughness.

In the impact experiments, intact eggshells are catapulted onto the ground at a high speed using a simple setup of rubber bands. The experimental setup provided a relatively high energy impact without the possibility of varying the imparted energy. The eggs are shot directly into a plastic bag touching the ground so that no fragments are lost for further evaluation.

In the explosion experiment initially the eggshell is flooded with hydrogen and hung vertically inside a plastic bag. The combustion reaction is initiated by igniting the escaping hydrogen on the top of the egg. The hydrogen immediately reacts with the oxygen which is also drawn up into the egg through the bottom hole, mixing with the remaining hydrogen. When enough air has entered to form a combustible mixture inside the egg, the flame back-fires through the top hole and starts a very quick exothermic reaction. The experiments are carried out inside a soft plastic bag so that no secondary fragmentations due to fragment-wall collisions occur; furthermore, no pieces were lost after explosion. Since the pressure that builds up during combustion can be slightly changed by the hole size, i.e., the smaller the hole, the higher the pressure at the explosion, we performed several series of experiments with hole diameters $d$ between 1.2 and $2.5 \mathrm{~mm}$. The limit values have practical reasons: a drilling nail of large diameter typically breaks the eggshell; on the other hand, it is extremely difficult to blow out an egg through a hole of diameter $1 \mathrm{~mm}$ or less.
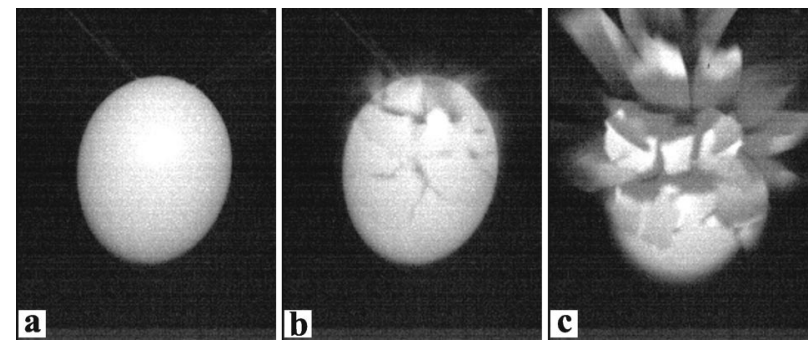

FIG. 1. Time evolution of the explosion of an eggshell, Consecutive snapshots taken by a high speed camera. The time difference between the snapshots is $0.001 \mathrm{~s}$.

It is possible to follow the time evolution of the explosion and impact processes by means of a high speed camera under well controlled conditions. Three consecutive snapshots of the explosion process are presented in Fig. 1 taken by a camera of $1000 \mathrm{~Hz}$ frequency. The ignition took place at the top of the egg in Fig. 1(a). The instant of backfiring and the initiation of combustion is captured in Fig. 1(b), while in Fig. 1(c) the flying pieces can already be seen. Based on the snapshots, the total duration of an explosion is estimated to be of the order of $1 \mathrm{~ms}$.

In the impact experiment the egg hits the ground in the direction of its longer axis, as is illustrated by the picture series of Fig. 2. After hitting the ground [Fig. 2(b)], the egg suffers gradual collapse as it moves forward [Figs. 2(c)2(h)], making the impact process relatively long compared to the explosion.

The resulting eggshell pieces are then carefully collected and placed on the tray of a scanner without overlap. In the scanned image, fragments are seen as black spots on a white background and were further analyzed by a cluster searching code. In the inset of Fig. 3 an example of scanned pieces of an impact experiment is shown where the broad variation of sizes can also be noticed with the naked eye. A dusty phase of shattered pieces [43] was also observed in the experiments with fragment sizes falling in the order of the pixel size of the scanner. The mass $m$ of fragments was determined as the number of pixels in the scanned image. Since shattered fragments were also comparable to normal dust pieces in the air,
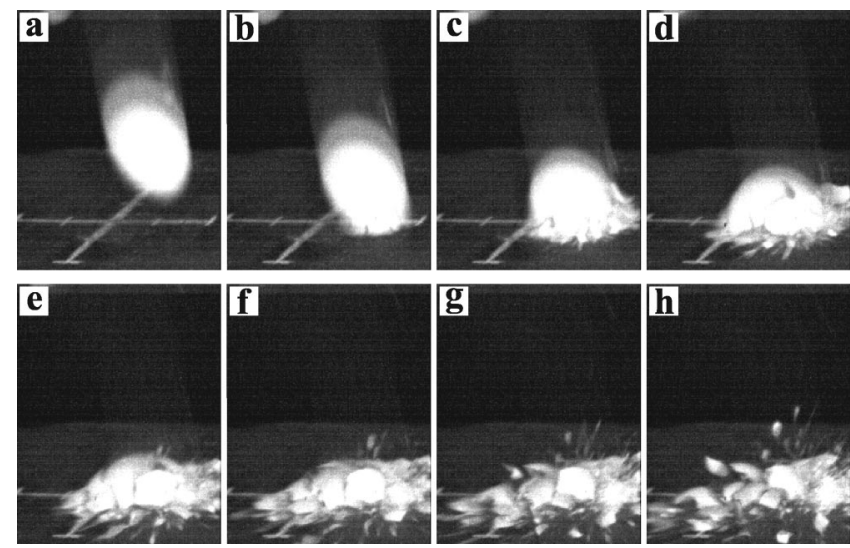

g
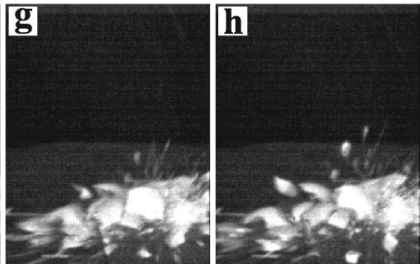

FIG. 2. Time series of the impact of an eggshell with the hard ground. The consecutive snapshots were taken by a high speed camera of $1 \mathrm{kHz}$ 


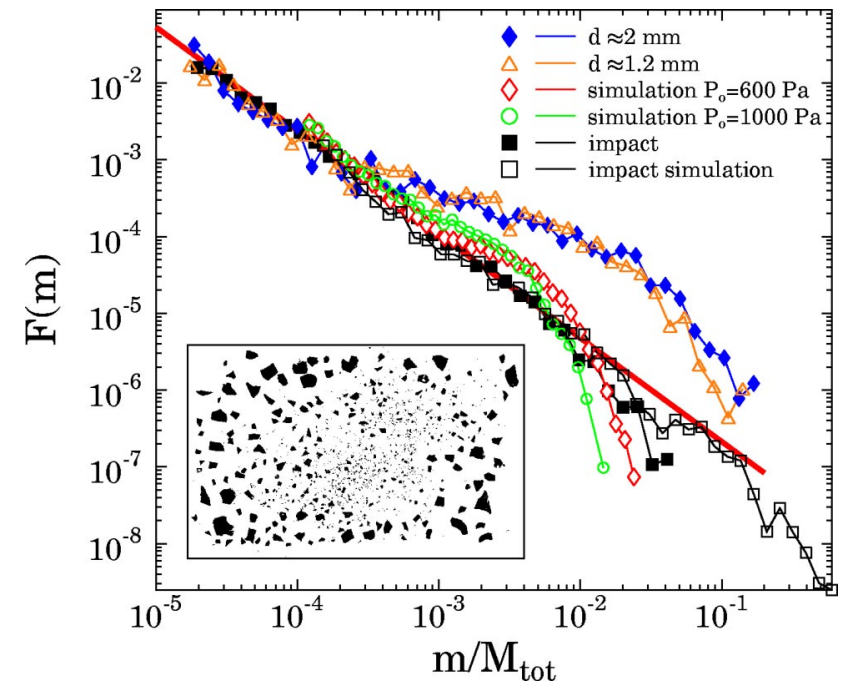

FIG. 3. (Color online) Comparison of fragment mass distributions obtained by explosion experiments with two hole sizes and the impact experiment to the simulation results. The inset shows a typical scanned set of fragments.

they were excluded in the analysis by setting the lower cutoff of fragment masses to a few pixels.

As the main quantitative result of the experiments we evaluated the mass distribution of fragments $F(m)$ which is defined so that $F(m) \Delta m$ provides the probability of finding a fragment with mass falling between $m$ and $m+\Delta m$. Figure 3 presents the fragment mass distributions $F(m)$ of brown eggs for impact and explosion experiments averaged over 10-20 eggshells for each curve. For the impact experiment, a power law behavior of the fragment mass distribution

$$
F(m) \sim m^{-\tau}
$$

can be observed over three orders of magnitude where the value of the exponent can be determined with high precision to $\tau=1.35 \pm 0.02$. Explosion experiments also result in a power law distribution of the same value of $\tau$ for small fragments with a relatively broad cutoff for the large ones. A smaller hole diameter $d$ in Fig. 3, i.e., higher pressure, gives rise to a larger number of fragments with a smaller cutoff mass and a faster decay of the distribution $F(m)$ for the large fragments. Comparing the number of fragments obtained, the ratio of the pressure values in the explosions at hole diameters $d=1.2$ and $2.0 \mathrm{~mm}$, presented in Fig. 3, was estimated to be about 1.6.

In order to investigate the effect of the material and size of the shell on the shape of the mass distribution $F(m)$, we carried out impact experiments with three different types of eggs, i.e., besides the above experiments with brown hen eggs, the impact experiment was repeated with white hen eggs and quail eggs. Due to differences in their chemical composition, white hen eggshells have different microscopic properties from the brown ones resulting in a significantly lower strength. Also, in addition to the different material, the volume of quail eggshells is about one-fourth of that of the hen eggs. Figure 4 shows that in spite of the differences of the shells, the mass distributions of their fragments have the

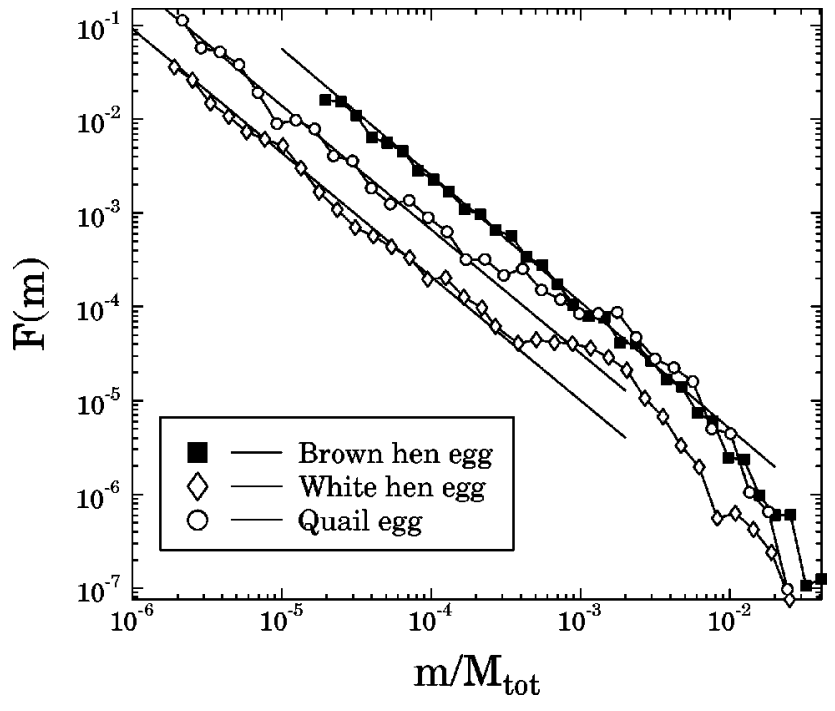

FIG. 4. Comparison of fragment mass distributions $F(m)$ obtained in impact experiments on brown and white hen eggs, and quail eggs.

same functional form. For small fragment masses the distributions can be well fitted by a power law with exponents $1.35 \pm 0.02,1.32 \pm 0.03$, and $1.32 \pm 0.03$, for brown and white hen eggs and quail eggs, respectively. The results of Figs. 3 and 4 demonstrate that the value of the exponent $\tau$ is independent of the size and microscopic material properties of the shells and of the way of loading.

It is important to note that the value of $\tau$ obtained for shell systems is significantly different from the experimental and theoretical results on fragmenting two-dimensional bulk systems where $1.5 \leqslant \tau \leqslant 2$ has been found $[2,7,8,13-17,22,23,35-38]$, and from three-dimensional ones where $\tau>2$ is obtained $[2,5,6,40,41]$.

\section{SIMULATIONS}

Most of the theoretical studies on fragmentation relay on large scale computer simulations since the capabilities of analytic approaches are rather limited in this field due to the complexity of the breakup process. Over recent years the discrete element method (DEM) has proven to be a very efficient numerical technique for fragmentation phenomena $[22,23,28,35-42]$ since it has the ability to handle large deformations arising dynamically, and naturally captures the propagation and interaction of a large number of simultaneously growing cracks, which is essential for fragmentation.

In order to investigate the fragmentation of spherical shells we constructed a three-dimensional discrete element model such that the surface of the unit sphere is discretized into randomly shaped triangles (Delaunay triangulation) by throwing points randomly and independently on the surface $[44,45]$. Based on the triangulation, the dual Voronoi tessellation of the surface is also carried out as illustrated in Fig. 5. The nodes of the triangulation represent pointlike material elements in the model whose mass is defined by the area of the Voronoi polygon assigned to them $[37,44,45]$. The bonds between nodes are assumed to be springs having linear elas- 


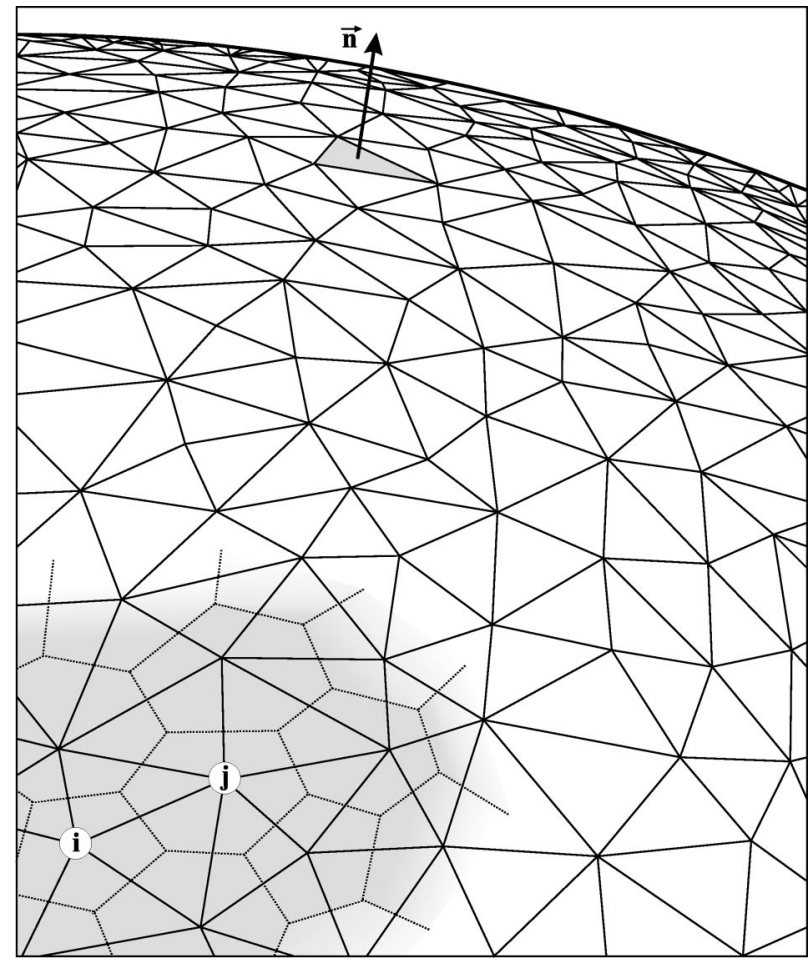

FIG. 5. Example of the Delaunay triangulation of a spherical surface. The dual Voronoi lattice is also shown in the lower left quadrant.

tic behavior up to failure. Disorder is introduced in the model solely by the randomness of the tessellation so that the mass assigned to the nodes and the length and cross section of the springs are determined by the tessellation (quenched structural disorder). After prescribing the initial conditions of a specific fragmentation process, the time evolution of the system is followed by solving the equation of motion of nodes by a predictor-corrector method of fourth order

$$
m_{i} \ddot{\vec{r}}_{i}=\vec{F}_{i}^{s}+\vec{F}_{i}^{\mathrm{ext}}+\vec{F}_{i}^{d}, \quad i=1, \ldots N
$$

where $\vec{F}_{i}^{s}$ is the sum of forces exerted by the springs connected to node $i$, and $\vec{F}_{i}^{\text {ext }}$ denotes the external driving force, which depends on the loading condition. To facilitate the relaxation of the system at the end of the fragmentation process, a small viscous damping force $\vec{F}_{i}^{d}$ was also introduced in Eq. (2).

In order to account for crack formation in the model, springs are assumed to break when their deformation $\varepsilon$ exceeds a certain breaking threshold $\varepsilon_{c}$. A fixed threshold value $\varepsilon_{c}=0.03$ is set for all the springs, resulting in a random sequence of breakings due to the disordered spring properties. The breaking criterion is evaluated at each iteration step and those springs which satisfy the condition are removed from the simulation. As a result of successive spring breakings cracks nucleate, grow, and merge on the spherical surface, which can give rise to a complete breakup of the shell into smaller pieces.

Fragments of the shell are defined in the model as sets of nodes (material elements) connected by the remaining intact
TABLE I. Parameter values used in the simulations.

\begin{tabular}{cccc}
\hline \hline Parameter & Symbol & Unit & Value \\
\hline Initial radius & $R$ & $\mathrm{~m}$ & 1 \\
Initial volume & $V_{0}$ & $\mathrm{~m}^{3}$ & 4.19 \\
Initial surface & $A_{0}$ & $\mathrm{~m}^{2}$ & 12.56 \\
Shell thickness & th & $\mathrm{m}$ & $5 \times 10^{-5}$ \\
Total mass & $M_{\text {tot }}$ & $\mathrm{kg}$ & 0.816 \\
Number of triangles & $N_{t}$ & & $\approx 44000$ \\
Number of nodes & $N_{n}$ & & $\approx 21000$ \\
Mass density & $\rho$ & $\mathrm{kg} / \mathrm{m}^{3}$ & 1300 \\
Time step & $\Delta t$ & $\mathrm{~s}$ & $3 \times 10^{-7}$ \\
Damping coefficient & $\gamma_{d}$ & $\mathrm{~kg} / \mathrm{s}$ & 0.1 \\
Spring Young modulus & $Y$ & $\mathrm{~N} / \mathrm{m}^{2}$ & $10^{9}$ \\
\hline \hline
\end{tabular}

springs. The process is stopped when the system has attained a relaxed state, i.e., when there is no spring breaking over a large number of iteration steps. The main advantage of the DEM is that it makes it possible to monitor a large number of microscopic physical quantities during the course of the simulation, which are hardly accessible experimentally, providing a deep insight into the fragmentation process. With the present computer capacities, DEM models can be designed to be realistic so that the simulation results can even complement the experimental information, extending our understanding. The most important parameter values used in our simulations are summarized in Table I.

In computer simulations two different ways of loading have been considered, which model the experimental conditions and represent limiting cases of energy input rates: (i) pressure pulse and (ii) impact load. A pressure pulse in a shell is introduced by imposing a fixed internal pressure $P_{0}$ from which the forces $\vec{F}_{j}^{\text {ext }}$ acting on the triangular surface elements are calculated as

$$
\vec{F}_{j}^{\text {ext }}=P_{0} A_{j} \vec{n}_{j},
$$

where $A_{j}$ denotes the actual area of triangle $j$ and the force points in the direction of the local normal $\vec{n}_{j}$; see also Fig. 5. The force $F_{j}^{\text {ext }}$ is equally shared by the three nodes of the triangle for which the equation of motion Eq. (2) is solved. Since the surface area of the shell increases, the expansion under constant pressure implies a continuous increase of the driving force and of the imparted energy.

The impact loading realizes the limiting case of instantaneous energy input by giving a fixed initial radially oriented velocity $v_{0}$ to the material elements and following the resulting time evolution of the system by solving the equation of motion Eq. (2). The control parameter of the system which determines the final outcome of the process are the fixed pressure $P_{0}$ and the initial kinetic energy $E_{0}$ for the pressure pulse and impact loading, respectively.

\section{THE BREAKUP PROCESS}

In the simulations, in both loading cases the spherical shell is initially completely stress-free with no energy stored 
in deformation. When a constant pressure is imposed, the total energy $E_{\text {tot }}$ of the shell increases due to the work done by the filling gas,

$$
E_{\mathrm{tot}}(V)=\int_{V_{0}}^{V} P_{0} d V=P_{0} \Delta V,
$$

where $V$ denotes the actual volume during the expansion and $\Delta V$ is the volume change with respect to the initial state $V_{0}$. The total energy can be written as the sum of the kinetic energy of material elements $E_{\text {kin }}$ and of the elastic energy $E_{\text {el }}$ stored in deformation, $E_{\mathrm{tot}}=E_{\mathrm{kin}}+E_{\mathrm{el}}$, where $E_{\mathrm{el}}$ is proportional to the change $\Delta A$ of surface area $A$ of the expanding sphere with respect to the initial surface $A_{0}$. Introducing the relative volume change $\Delta v=\Delta V / V_{0}$ as an independent variable, the total energy and the elastic energy can be cast in the forms

$$
\begin{gathered}
E_{\mathrm{tot}}=P_{0} V_{0} \Delta v, \\
E_{\mathrm{el}}=C\left[(\Delta v+1)^{1 / 3}-1\right]^{2},
\end{gathered}
$$

where the surface change $\Delta A$ is expressed in terms of $\Delta v$. Furthermore, the parameter $C$ of the system depends on the properties of the triangulation and the characteristic physical quantities of springs (Young modulus, length, thickness). It is interesting to note that a specific pressure value $P_{0}^{*}$ exists, below which the expansion always stops at a maximum volume change $\Delta v_{\max }$ depending on $P_{0}$; however, for $P_{0}>P_{0}^{*}$ the expansion always keeps accelerating. For a given $P_{0}$ $<P_{0}^{*}$ the value of $\Delta v_{\max }$ can be determined from the condition $E_{\mathrm{tot}}=E_{\mathrm{el}}$ so that

$$
P_{0} V_{0} \Delta v_{\max }=C\left[\left(\Delta v_{\max }+1\right)^{1 / 3}-1\right]^{2},
$$

and $P_{0}^{*}$ can be identified as the highest pressure for which Eq. (7) can be solved for $\Delta v_{\max }$. Usually $\Delta v_{\max }$ can only be realized at low pressure values, because at higher pressures the system suffers complete breakup much below $\Delta v_{\max }$, due to the finite strength of the springs. Figure 6 illustrates the evolution of the total $E_{\mathrm{tot}}$, kinetic $E_{\mathrm{kin}}$, and elastic $E_{\mathrm{el}}$ energies as a function of $\Delta v$ for both pressure and impact loading. In the case of pressure loading it can be observed that the total energy $E_{\text {tot }}$ extracted from the simulations agrees well with the analytic prediction of Eq. (5). The numerical value of the multiplication factor $C$ of the elastic energy was obtained by fitting the expression Eq. (6) to the curve of $E_{\mathrm{el}}(\Delta v)$ in the figure. Due to the constant pressure, the total force $F$ acting on the shell is proportional to the actual surface area $F$ $\sim A P_{0}$ so that the system is driven by an increasing force during the expansion process. Since the driving force $F$ increases with a diminishing rate when approaching the limit volume change $\Delta v_{\max }$, it follows that the pressure loading case is analogous to the stress controlled quasistatic loading of bulk specimens. According to the simulations, under pressure loading there exists a critical pressure $P_{c}$ below which the expansion always stops at a finite volume and the shell only suffers partial failure (damage) in the form of cracks but keeps its integrity. When the pressure exceeds $P_{c}$, however, the system surpasses the critical volume change $\Delta v_{c}$ when a large amount of springs abruptly break resulting in the

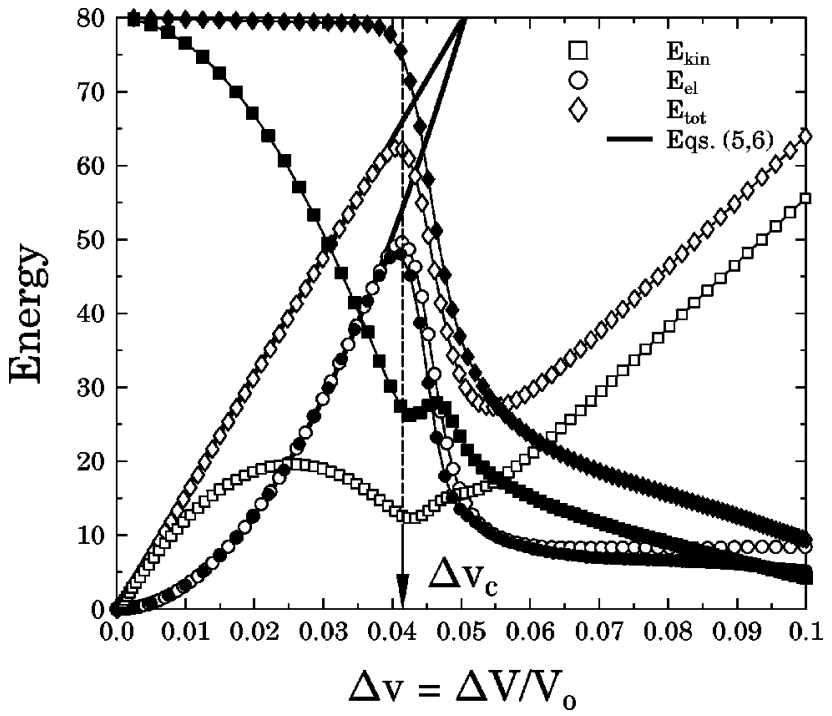

FIG. 6. The kinetic $E_{\text {kin }}$, elastic $E_{\mathrm{el}}$, and total $E_{\mathrm{tot}}$ energies as a function of the relative volume change $\Delta v$. Open symbols stand for expansion under constant pressure while the filled ones characterize the impact loading. The sudden drop of the total and elastic energy at $\Delta v_{c}$ indicates the rapid breakup of the system. For $E_{\text {tot }}$ of the pressure loading the thick solid line follows Eq. (5), while for $E_{\mathrm{el}}$ the function given by Eq. (6) was fitted with $C=312000$ as a parameter.

breakup of the system (fragmentation). Note that $P_{c} \ll P_{0}^{*}$.

The critical volume change $\Delta v_{c}$, where fragmentation sets in during the expansion, can be identified by the location of the sudden drop of the elastic energy in Fig. 6 caused by the large amount of spring breaking which occurs in a very narrow $\Delta v$ interval, resulting in a rapid formation of cracks on the surface. The value of $\Delta v_{c}$ is mainly determined by the fixed breaking threshold $\varepsilon_{c}$ and the disordered spring properties. Since the shell is under constant pressure the nucleated microcracks can grow and join giving rise to planar pieces surrounded by a free crack surface (fragment), as is illustrated in Figs. 7(c)-7(e). First large fragments are formed which then break up into smaller pieces until the surviving springs can sustain the remaining stress; see Figs. 7(c)-7(e). For simplicity, in the simulations the pressure is kept constant even if the system has lost its integrity, which formally has the consequence that pieces of the shell formed in the final state of fragmentation keep accelerating under the action of a constant force, which explains the increasing kinetic energy $E_{\text {kin }}$ in Fig. 6 following fragmentation. The volume of the system is numerically calculated as the sum of the volume of pyramidal objects defined by the surface elements and the center of the sphere, which provides a meaningful result even after breakup in Fig. 6 in the vicinity of $\Delta v_{c}$. The critical pressure $P_{c}$, required to exceed the critical volume change $\Delta v_{c}$ to achieve fragmentation, can be estimated as $P_{c}=E_{\mathrm{el}}\left(\Delta v_{c}\right) /\left(V_{0} \Delta v_{c}\right)$.

When loading is imposed by an instantaneous energy input $E_{0}$, there is no further energy supply, and the total energy of the system is either constant or decreases due to the viscous dissipation and the breaking of springs (see Fig. 6). Since the elastic energy $E_{\mathrm{el}}$ is solely determined by the de- 
a)
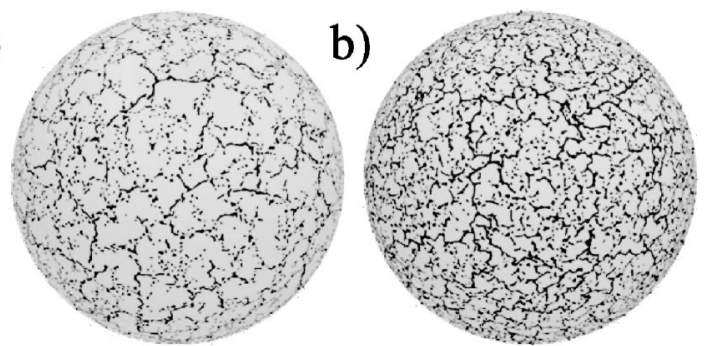

c)

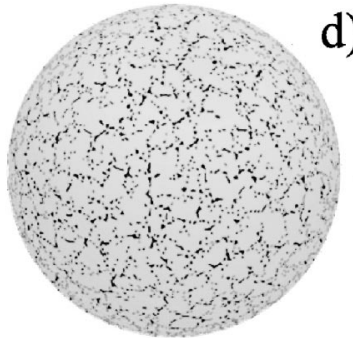

d)

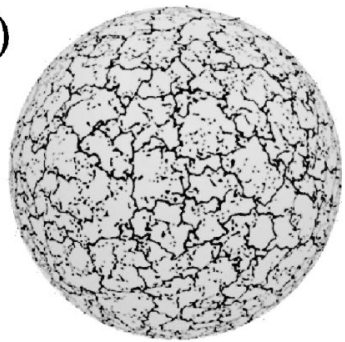

e)
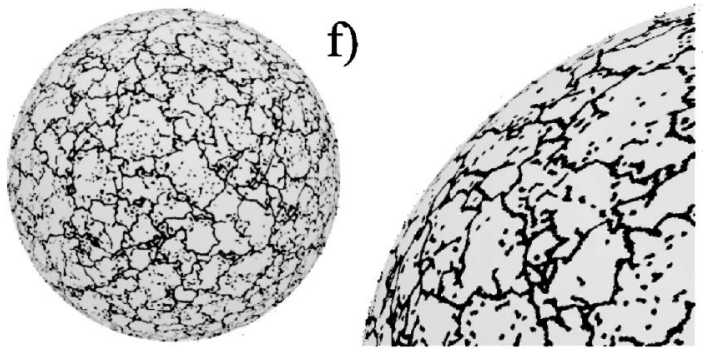

FIG. 7. Cracks on the shell surface. Final states of impact experiments at energies $E_{0} / E_{c} \approx 0.8$ (a) and $2.8(\mathrm{~b})$. Time evolution of the cracking process under a constant pressure of $P_{0} / P_{c} \approx 4.0$ (c), (d) until the final relaxed state is reached (e), with a magnified view of fragments (f). Particle positions are projected back to their initial states on the surface. Fragments are identified as shell pieces surrounded by cracks.

formation, the curve of $E_{\mathrm{el}}$ and the critical volume change $\Delta v_{c}$ where breakup arises in Fig. 6 coincide with the corresponding values of the pressure loading. Similarly to the pressure loading case, simulations revealed that a critical value of the imparted energy $E_{c}$ can be identified below which the shell maintains its integrity, suffering only damage, while exceeding $E_{c}$ gives rise to a complete fragmentation of the shell. The resulting fragments on the shell surface obtained in the fragmented regime can be seen in Figs. 7(a) and $7(b)$.

\section{FRAGMENT MASSES}

To give a quantitative characterization of the breakup of shells and to reveal the nature of the transition between the damaged and fragmented states, large scale simulations have been performed varying the control parameters, i.e., the fixed pressure $P_{0}$ and the imparted energy $E_{0}$ over a broad range. The most important characteristics of our fragmenting shell system that can be compared to the experimental findings is the variation of fragment masses when changing the control parameters. In the simulations two cutoffs arise for the fragment masses, where the lower one is defined by the single unbreakable material elements of the model and the upper one is due to the finite size of the system.

For both types of loading above the critical point the typical fragment size obtained at the instant of breakup decreases with increasing control parameter, which can be described analytically in terms of an energy balance argument similarly to the one given in Ref. [27]. The loading energy of a shell region of linear extension $L$ and mass $m \sim L^{2}$, i.e., the energy stored in the motion of particles separating the piece from its surroundings, can be written as $\left[m / M_{\text {tot }}\right] E_{\text {kin }}\left(\Delta v_{c}\right) L^{2}$ $=\left[E_{\mathrm{kin}}\left(\Delta v_{c}\right) / M_{\mathrm{tot}}\right] L^{4}$, where $E_{\mathrm{kin}}\left(\Delta v_{c}\right)$ denotes the total kinetic energy of the shell at the instant of breakup and $M_{\text {tot }}$ is the total mass of the shell. The separation of the piece from its surroundings costs energy proportional to the fragment surface $\sim L$. The equilibrium fragment size can be obtained by minimizing the sum of the loading and surface energy densities $\rho_{E}$ :

$$
\rho_{E} \sim \frac{E_{\mathrm{kin}}\left(\Delta v_{c}\right)}{M_{\mathrm{tot}}} L^{2}+\frac{1}{L}
$$

with respect to $L$, which results in $L \sim E_{\text {kin }}^{-1 / 3}$. It has been shown in the previous section that at the critical point $P_{c}, E_{c}$ the total kinetic energy of the system when breakup occurs takes zero value $E_{\text {kin }}\left(\Delta v_{c}\right)=0$. It follows that above the critical point $E_{\text {kin }}$ has a linear dependence on the distance from the critical point so that $E_{\mathrm{kin}}\left(\Delta v_{c}\right) \sim\left(P_{0}-P_{c}\right)$ for $P_{0}>P_{c}$, and $E_{\text {kin }}\left(\Delta v_{c}\right) \sim\left(E_{0}-E_{c}\right)$ for $E_{0}>E_{c}$ hold. Substituting these results into Eq. (8), the typical fragment mass at the instant of breakup can be cast into the form

$$
\begin{aligned}
& m \sim\left(P_{0}-P_{c}\right)^{-2 / 3} \text { for } P_{0}>P_{c}, \\
& m \sim\left(E_{0}-E_{c}\right)^{-2 / 3} \text { for } E_{0}>E_{c} .
\end{aligned}
$$

Equations (9) and (10) express that the typical fragment mass obtained at the time of breakup decreases according to a power law with increasing distance from the critical point. The exponent of the power law is universal in the sense that it does not depend on specific material properties of the shell. Later on during the fragmentation process the elastic energy stored in deformation may result in successive breakings of the large fragments. Hence, it can be expected that Eqs. (9) and (10) describe the scaling behavior of the largest fragments, which did not undergo substantial size reduction until reaching the final relaxed state.

\section{A. Largest fragments}

To characterize the degree of fragmentation, i.e., the size reduction achieved in the simulations, we calculated the average mass of the largest $\left\langle M_{\max } / M_{\text {tot }}\right\rangle$ and of the second largest $\left\langle M_{\max }^{2 \text { nd }} / M_{\text {tot }}\right\rangle$ fragments normalized by the total mass as a function of the pressure $P_{0}$ and input energy $E_{0}$ in the case of pressure and impact loading, respectively $[35,36]$. The results are presented in Figs. 8 and 9. It can be seen that in both cases the maximum fragment mass is a monotonically decreasing function of the control parameters $P_{0}$ and $E_{0}$; however, the functional forms are different in the two cases. Low pressure values in Fig. 8 result in a breaking of springs; 


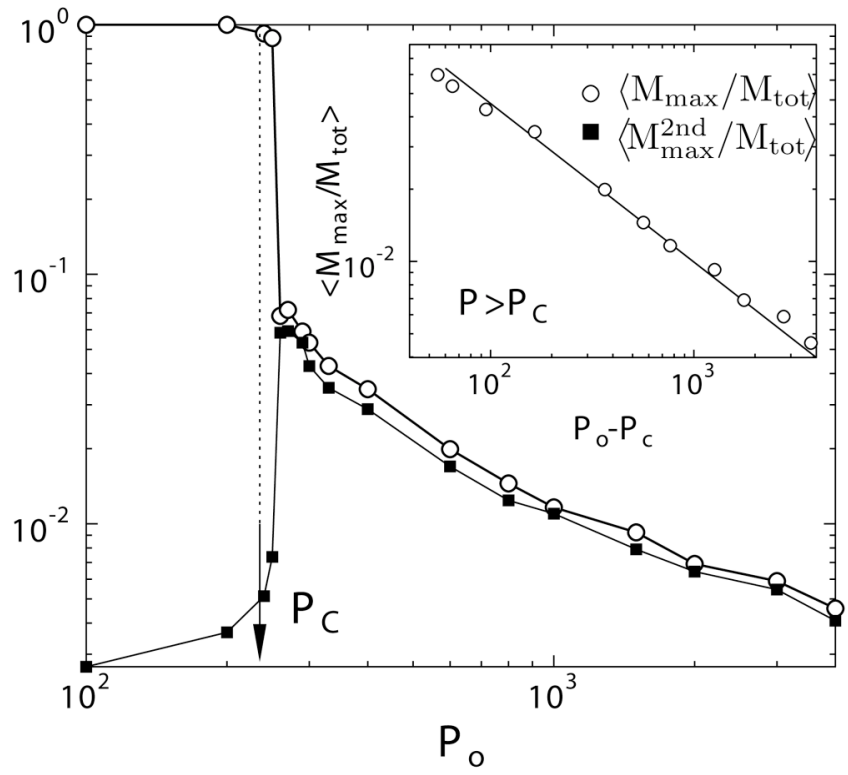

FIG. 8. Average mass of the largest $\left\langle M_{\max } / M_{\text {tot }}\right\rangle$ and second largest $\left\langle M_{\max }^{2 \text { nd }} / M_{\text {tot }}\right\rangle$ fragment normalized by the total mass as a function of imposed pressure $P_{0}$. The inset presents a log-log plot of $\left\langle M_{\max } / M_{\text {tot }}\right\rangle$ as a function of the distance from the critical point $P_{0}-P_{c}$. In the main figure the value of $P_{c}$ is indicated by an arrow.

however, hardly any fragments are formed except for single elements broken out of the shell along cracks. Hence, the mass of the largest fragment is practically equal to the total mass $M_{\text {tot }}$ of the system, while the second largest fragment is orders of magnitude smaller (damage). However, when increasing the pressure above the threshold value $P_{c}$ the largest fragment mass becomes much smaller than the total mass; furthermore, in this regime there is only a slight difference

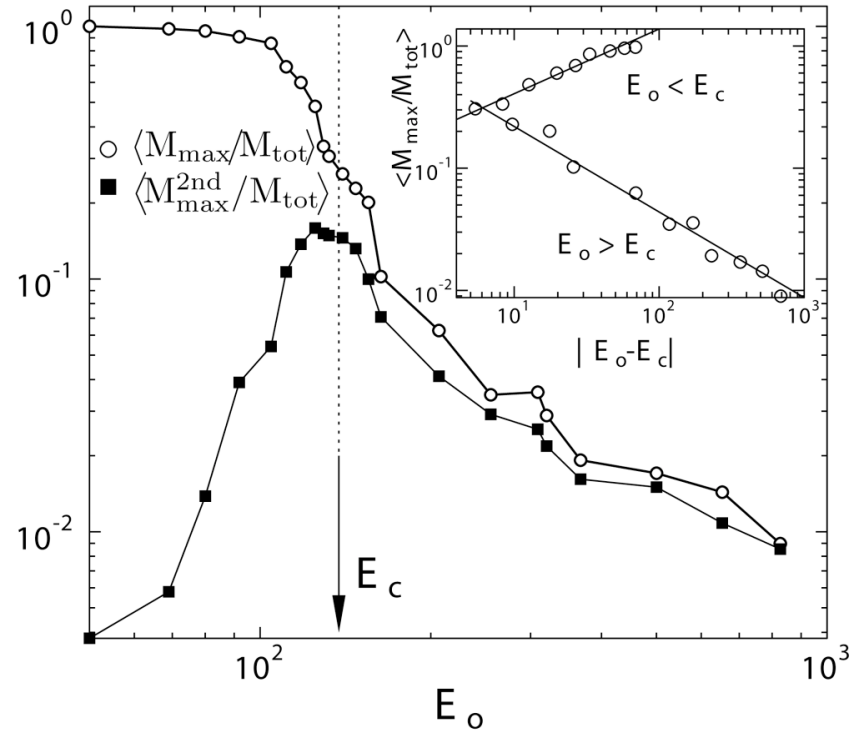

FIG. 9. $\left\langle M_{\max } / M_{\text {tot }}\right\rangle$ and $\left\langle M_{\max }^{2 \text { nd }} / M_{\text {tot }}\right\rangle$ as a function of the imparted energy. The inset presents a log-log plot of the largest mass as a function of $\left|E_{0}-E_{c}\right|$, where the increasing and decreasing branches characterize the damaged and fragmented states, respectively. The location of $E_{c}$ is indicated in the main figure. between the largest and second largest fragments, indicating the complete disintegration of the shell into pieces (fragmentation). The value of the critical pressure $P_{c}$ needed to achieve fragmentation and the functional form of the curve of $\left\langle M_{\max } / M_{\text {tot }}\right\rangle$ above $P_{c}$ was determined such that $\left\langle M_{\text {max }} / M_{\text {tot }}\right\rangle$ was plotted as a function of the difference $\mid P_{0}$ $-P_{c} \mid$ varying $P_{c}$ until a straight line is obtained on a double logarithmic plot. The result is presented in the inset of Fig. 8 where a power law dependence of $\left\langle M_{\text {max }} / M_{\text {tot }}\right\rangle$ is evidenced as a function of the distance from the critical point:

$$
\left\langle M_{\max } / M_{\text {tot }}\right\rangle \sim\left|P_{0}-P_{c}\right|^{-\alpha} \text { for } P_{0}>P_{c} .
$$

The exponent $\alpha=0.66 \pm 0.02$ was obtained in good agreement with the analytic prediction of Eq. (9). Detailed studies in the vicinity of $P_{c}$ revealed a finite jump of both $\left\langle M_{\max } / M_{\text {tot }}\right\rangle$ and $\left\langle M_{\max }^{2 \text { nd }} / M_{\text {tot }}\right\rangle$ at $P_{c}$ which implies that fragmentation occurs as an abrupt transition at the critical point, see Fig. 8.

In Fig. 9 the corresponding results are presented for the case of impact loading as a function of the total energy $E_{0}$ imparted to the system initially. The mass of the largest fragment is again a monotonically decreasing function of the control parameter; however, it is continuous in the entire energy range considered. Careful analyses revealed the existence of two regimes with a continuous transition at a critical value of the imparted energy $E_{c}$. In the inset of Fig. 9 $\left\langle M_{\max } / M_{\mathrm{tot}}\right\rangle$ is shown as a function of the distance from the critical point $\left|E_{0}-E_{c}\right|$ where $E_{c}$ was determined using the same technique as for $P_{c}$. Contrary to the pressure loading, $\left\langle M_{\text {max }} / M_{\text {tot }}\right\rangle$ exhibits a power law behavior on both sides of the critical point but with different exponents,

$$
\begin{aligned}
& \left\langle M_{\max } / M_{\text {tot }}\right\rangle \sim\left|E_{0}-E_{c}\right|^{\beta} \text { for } E_{0}<E_{c}, \\
& \left\langle M_{\text {max }} \mid M_{\text {tot }}\right\rangle \sim\left|E_{0}-E_{c}\right|^{-\alpha} \text { for } E_{0}>E_{c} .
\end{aligned}
$$

The numerical values of the exponents were obtained as $\alpha$ $=0.66 \pm 0.02$ and $\beta=0.5 \pm 0.02$, above and below the critical point, respectively. Note that the value of $\alpha$ coincides with the corresponding exponent of the pressure loading and is in good agreement with the analytic prediction of Eq. (10). Below the critical point the second largest fragment is again orders of magnitude smaller than the largest one, which implies that in this energy range the shell suffers only damage in the form of cracks, while above the critical point the breakup of the entire shell results in comparable values of the largest and second largest fragment masses. At the transition point $E_{c}$ between the damaged and fragmented states the mass of the second largest fragment has a maximum, while the curve of the largest one exhibits a curvature change (see Fig. 9).

\section{B. Average fragment mass}

More insight can be obtained into the fragmentation process by studying the so-called single-event moments of fragment masses 


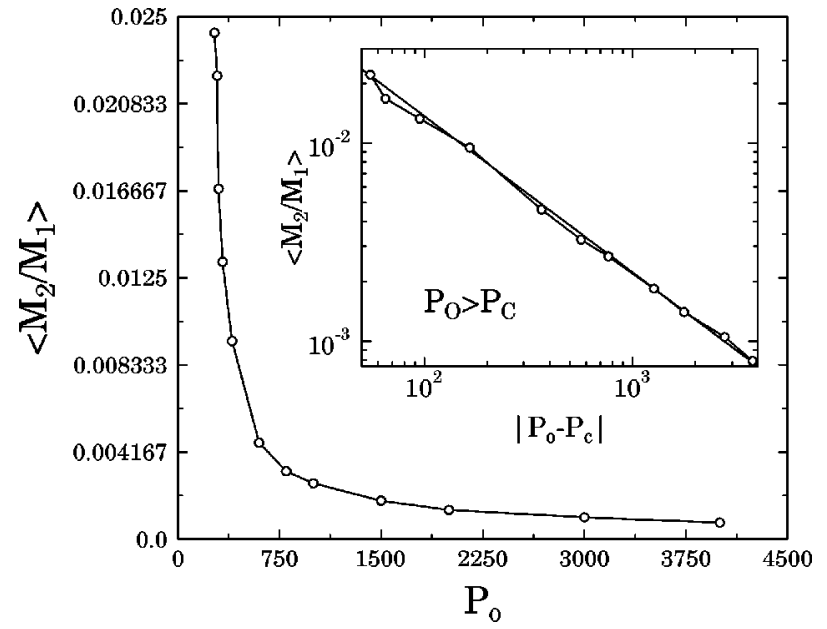

FIG. 10. The average fragment mass as a function of the imposed pressure $P_{0}$. The inset presents a log-log plot of the average mass as a function of the distance from the critical point $P_{0}-P_{c}$ for pressure values $P_{0}>P_{c}$. The value of $P_{c}$ is the same as in Fig. 8 .

$$
M_{k}^{j}=\sum_{m} m^{k} n^{j}(m)-M_{\max }^{k},
$$

where $M_{k}^{j}$ denotes the $k$ th moment of fragment masses $m$ in the $j$ th realization of a fragmentation process, and $n^{j}(m)$ is the number of fragments of mass $m$ in event $j$. The ratio of the second $M_{2}^{j}$ and the first $M_{1}^{j}$ moments provides a measure for the average fragment mass in a specific experiment $j$ :

$$
\bar{M}^{j}=\frac{M_{2}^{j}}{M_{1}^{j}} .
$$

Averaging over simulations with different realizations of disorder, the average fragment mass $\bar{M}=\left\langle M_{2}^{j} / M_{1}^{j}\right\rangle$ was obtained as a function of the control parameter of the system.

Due to the abrupt nature of the transition from the damaged to the fragmented states at the critical pressure, $\bar{M}$ cannot be evaluated below $P_{c}$ under pressure loading. However, when $P_{0}$ exceeds the critical pressure $P_{c}$ the average fragment mass monotonically decreases in Fig. 10. The inset of Fig. 10 shows $\bar{M}$ as a function of the distance from the critical point $\left|P_{0}-P_{c}\right|$ where the same value of $P_{c}$ was used as in Fig. 8. A power law dependence of $\bar{M}$ is evidenced as a function of $\left|P_{0}-P_{c}\right|$ :

$$
\bar{M} \sim\left|P_{0}-P_{c}\right|^{-\gamma}
$$

for $P_{0}>P_{c}$ and the value of the exponent was obtained to be $\gamma=0.8 \pm 0.02$. For impact loading $\bar{M}$ can be evaluated on both sides of the critical point with a sharp peak in the vicinity of $E_{c}$ which is typical for continuous phase transitions in finite systems (see Fig. 11). A power law dependence of $\bar{M}$ on the distance from the critical point,

$$
\bar{M} \sim\left|E_{0}-E_{c}\right|^{-\gamma},
$$

is again revealed for $E_{0}>E_{c}$, which is illustrated in the inset of Fig. 11. The value of the exponent was determined by

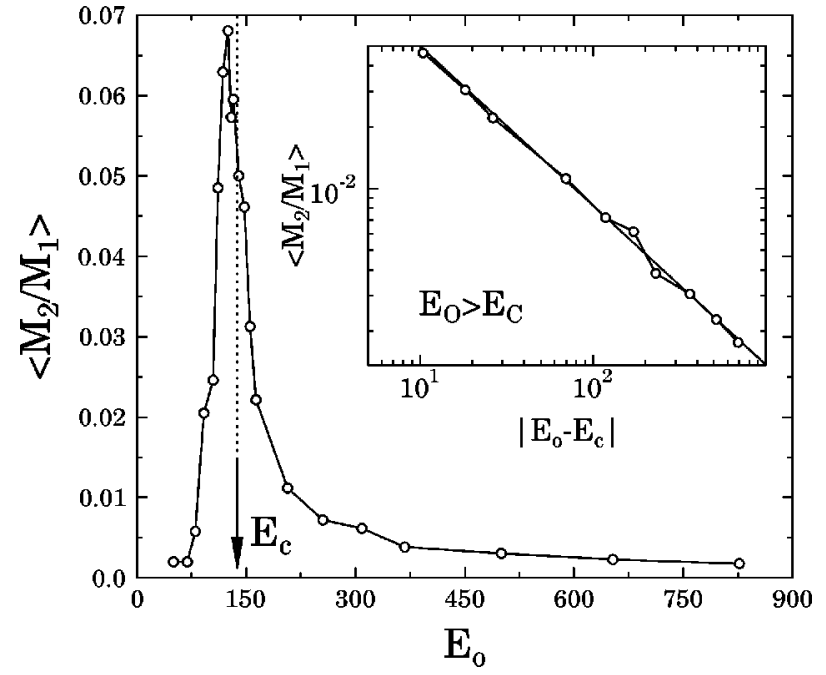

FIG. 11. The average fragment mass $\left\langle M_{2} / M_{1}\right\rangle$ as a function of the imparted energy $E_{0}$. The two regimes can be clearly distinguished. The location of the critical point proved to be exactly the same as in Fig. 9. The inset shows a log-log plot of the average mass as a function of $\left|E_{0}-E_{c}\right|$.

fitting $\gamma=0.79 \pm 0.02$, which practically coincides with the $\gamma$ value of pressure loading.

\section{Fragment mass distributions}

The most important characteristic quantity of our system which can also be compared to the experimental results is the mass distribution of fragments $F(m)$. Under impact loading for $E_{0}<E_{c}$ we found that $F(m)$ has a pronounced peak at large fragments indicating the presence of large damaged pieces (see Fig. 12). Approaching the critical point $E_{c}$ the peak gradually disappears and the distribution asymptotically becomes a power law at $E_{c}$. We can observe in Fig. 12 that above the critical point the power law remains for small frag-

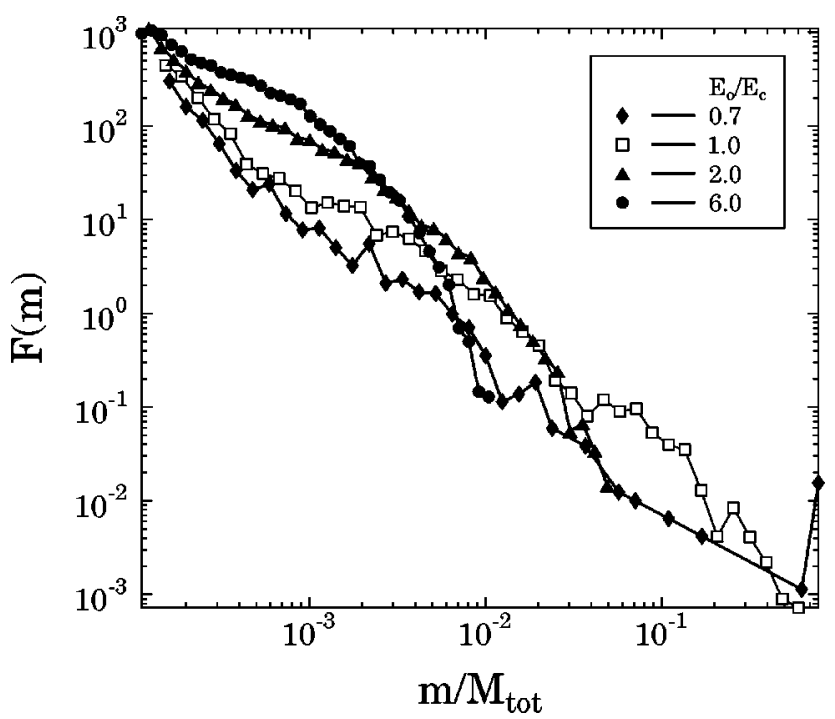

FIG. 12. Mass distribution of fragments at various energies below and above the critical point. 


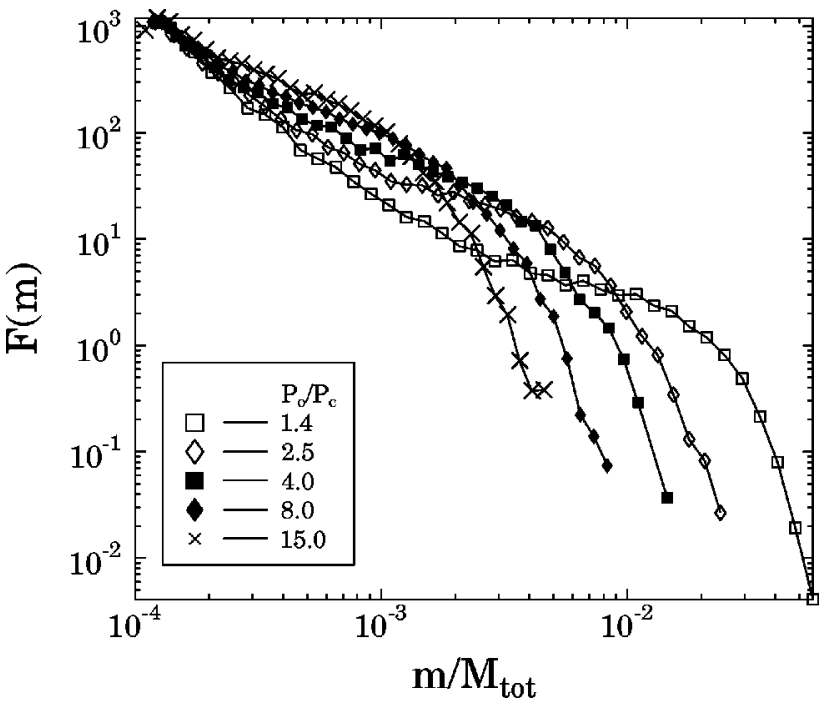

FIG. 13. Mass distribution of fragments at various pressure values.

ments, followed by a cutoff for the large ones, which decreases with increasing $E_{0}$.

For pressure loading $F(m)$ can only be evaluated above $P_{c}$. The evolution of $F(m)$ with increasing pressure is presented in Fig. 13, where the mass distribution always shows a power law behavior for small fragments with a relatively broad cutoff for the large ones. For the purpose of comparison, a mass distribution $F(m)$ obtained at an impact energy close to the critical point $E_{c}$, and distributions at two different pressure values $P_{0}$ of the ratio 1.6, are plotted in Fig. 3 along with the experimental results. Excellent agreement with the experimental and theoretical results is evidenced for impact loading. For pressure loading, the functional form of $F(m)$ has a nice qualitative agreement with the experimental findings on the explosion of eggs; furthermore, distributions at the same ratio of pressure values obtained by simulations and experiments show the same tendency of evolution (see Fig. 3).

Figures 14 and 15 demonstrate that by rescaling the mass distributions above the critical point by plotting $F(m) \bar{M}^{\delta}$ as a function of $m / \bar{M}$ an excellent data collapse is obtained with $\delta=1.6 \pm 0.03$. The data collapse implies the validity of the scaling form

$$
F(m) \sim m^{-\tau} f(m / \bar{M}),
$$

typical for critical phenomena. The cutoff function $f$ has a simple exponential form $\exp (-m / \bar{M})$ for impact loading (see Fig. 14), and a more complex one containing also an exponential component for the pressure case (see Fig. 15). The average fragment mass $\bar{M}$ occurring in the scaling form Eq. (18) diverges according to a power law given by Eqs. (16) and (17) when approaching the critical point. The good quality of collapse and the functional form Eq. (18) also imply that the exponent $\tau$ of the mass distribution does not depend on the value of the pressure $P_{0}$ or the kinetic energy $E_{0}$

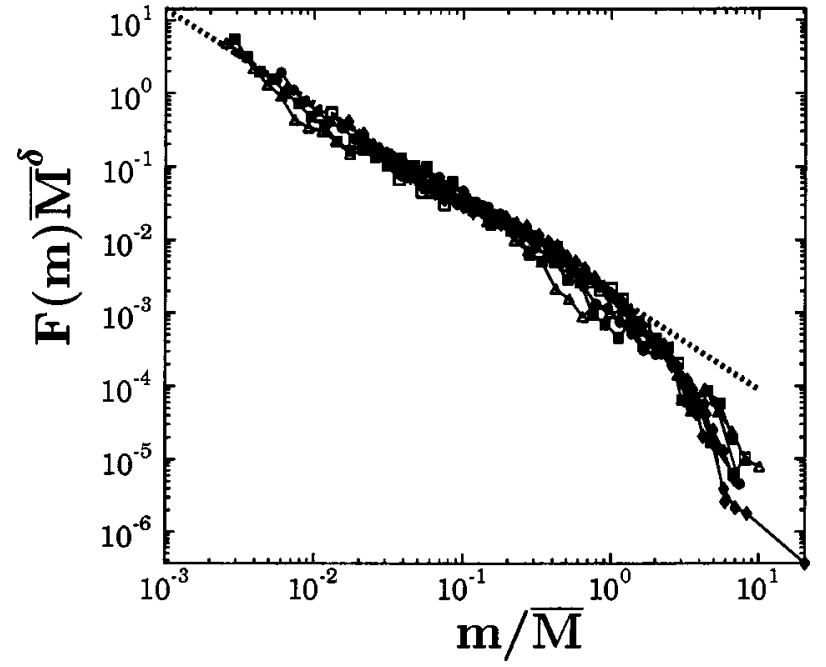

FIG. 14. Rescaled plot of the mass distributions for imparted energies above the critical point $E_{0}>E_{c}$. The dashed line shows the fitted power law with an exponent $\tau=1.35 \pm 0.03$.

contrary to the bulk fragmentation where an energy dependence of $\tau$ was reported [29].

The rescaled plots make possible an accurate determination of the exponent $\tau$, where $\tau=1.35 \pm 0.03$ and $\tau$ $=1.55 \pm 0.03$ were obtained for impact and pressure loading, respectively. Hence, a good quantitative agreement of the theoretical and experimental values of the exponent $\tau$ is evidenced for the impact loading of shells; however, for the case of pressure loading the numerically obtained exponent turned out to be somewhat higher than in the case of exploded eggs.

\section{DISCUSSION AND OUTLOOK}

We presented a detailed experimental and theoretical study of the breakup of closed shells. For the purpose of

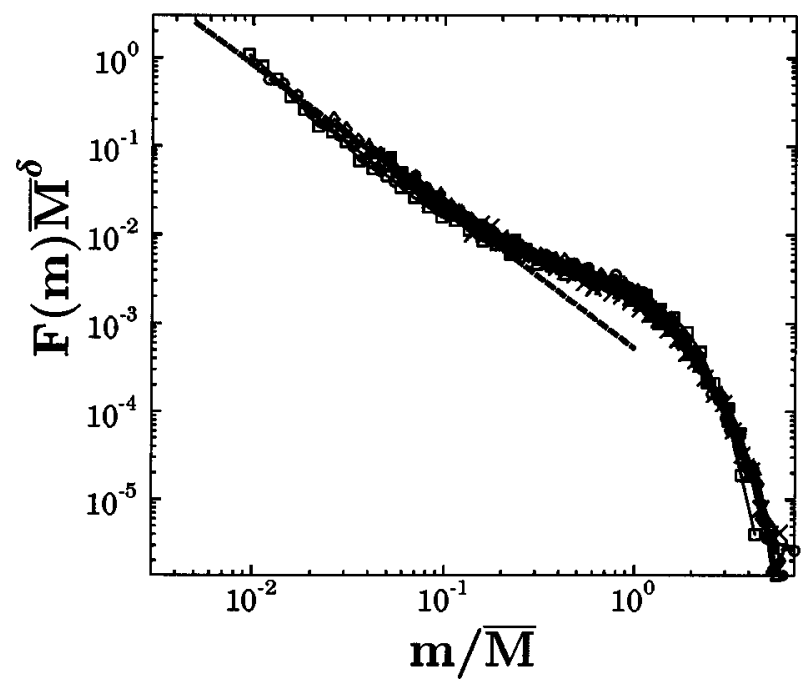

FIG. 15. Rescaled plot of the mass distributions of various pressure values above the critical point $P_{0}>P_{c}$. The dashed line indicates the fitted power law with an exponent $\tau=1.55 \pm 0.03$. 
experiments brown and white hen eggshells and quail eggshells were carefully prepared to ensure a high degree of brittleness of the disordered shell material. The breakup of the shell was studied under two different loading conditions, i.e., explosion caused by a combustible mixture and impact with the hard ground. As the main outcome of the experiments, the mass distribution of fragments proved to be a power law in both loading cases for small fragment sizes; however, qualitative differences were obtained in the limit of large fragments for the shape of the cutoff. Experiments on eggshells with different material properties and overall size result in fragment mass distributions of the same functional form with practically the same value of the exponent $\tau$, which indicates the universality of shell fragmentation.

We worked out a discrete element model for the breakup of shells which provides an insight into the dynamics of the process by simultaneously monitoring several microscopic quantities in the framework of molecular dynamics simulations. In the simulations two ways of loading have been considered, which mimic the experimental conditions and represent limiting cases of energy input rates: during an expansion under constant pressure $P_{0}$ the shell is driven by an increasing force with a continuous increase of the imparted energy, while the impact loading realizes the instantaneous input of the energy $E_{0}$. Simulations revealed that, depending on the value of $P_{0}$ and $E_{0}$, the final outcome of the breakup process can be classified into two states, i.e., damaged and fragmented, with a sharp transition between at a critical value of the control parameters $P_{c}$ and $E_{c}$. In the fragmented regime, power law fragment mass distributions were obtained in satisfactory agreement with the experimental findings. Analyzing the behavior of the system in the vicinity of the critical point $P_{c}, E_{c}$, we showed that power law distributions arise in the breakup of shells due to an underlying phase transition between the damaged and fragmented states, which proved to be abrupt for explosion, and continuous for impact. The parameters characterizing the shell in the computer simulations were not fitted to any specific material. The qualitative and quantitative agreement of the experimental and theoretical results further supports the universality of the fragmentation of shell systems.
Due to their unique characteristics, the breakup of shells defines a universality class of fragmentation phenomena that is different from that of two- and three-dimensional bulk systems. The local geometry of shell structures is two dimensional; however, their dynamics proceeds in the embedding three-dimensional space, which allows for a rich variety of failure modes not present in bulk solids. This effective dimensionality of the fragmenting system plays a crucial role in the generation of cracks, which finally determines the exponent of the fragment mass distribution. The effect of the embedding spatial dimension on the dynamics of fragmentation has also been recently discussed in Ref. [46]. Based on universality, our results should be applicable to describe the breakup of other closed shell systems composed of disordered brittle materials. Explosions of shell-like fuel containers, tanks, and high pressure vessels often occur as accidental events in industry, or in space missions, where also the explosion of complete satellites may occur, creating a high amount of space debris orbiting Earth. For the safety design of shell constructions and for the tracking of space debris, it is crucial to have a comprehensive understanding of the breakup of shells. Due to the universality of fragmentation phenomena, our results can be used for these purposes.

In the fragmentation of bulk systems under appropriate conditions a so-called detachment effect is observed when a surface layer breaks off from the bulk and undergoes a separate fragmentation process $[29,37]$. This effect also shows up in the fragment mass distributions in the form of a power law regime of small fragments of an exponent smaller than for the large ones. Our results on shell fragmentation can also provide a possible explanation of this kind of composite power law of bulk fragmentation $[29,37]$.

\section{ACKNOWLEDGMENTS}

This work was supported by the Collaborative Research Center SFB381 and by OTKA Grants No. T037212 and No. M041537. F.K. was supported by the Research Contract FKFP 0118/2001 and by the György Békési Foundation of the Hungarian Academy of Sciences. The authors are also thankful for the technical support of H. Gerhard from IKP of the University of Stuttgart.
[1] Fragmentation Phenomena, edited by D. Beysens, X. Campi, and E. Pefferkorn (World Scientific, Singapore, 1995).

[2] D. L. Turcotte, J. Geophys. Res., [Space Phys.] 91, 1921 (1986).

[3] L. Zhang, X. Jin, and H. He, J. Phys. D 32612 (1999).

[4] J. J. Gilvarry, J. Appl. Phys. 32, 391 (1961). 32, 400 (1961)

[5] T. Matsui, T. Waza, K. Kani, and S. Suzuki, J. Geophys. Res. B 87, 10968 (1982).

[6] A. Fujiwara and A. Tsukamoto, Icarus 44, 142 (1980).

[7] H. Inaoka, E. Toyosawa, and H. Takayasu, Phys. Rev. Lett. 78, 3455 (1997).

[8] T. Kadono, Phys. Rev. Lett. 78, 1444 (1997).

[9] T. Kadono and M. Arakawa Phys. Rev. E 65, 035107 (2002).
[10] H. Inaoka, M. Ohno, Fractals 11, 369 (2003).

[11] F. G. Bridges, A. Hatzes, and D. N. C. Lin, Nature (London) 309, 333 (1984).

[12] N. Arbiter, C. C. Harris and G. A. Stamboltzis, Trans. Soc. Min. Eng. AIME 244, 119 (1969).

[13] L. Oddershede, P. Dimon, and J. Bohr, Phys. Rev. Lett. 71, 3107 (1993).

[14] A. Meibom and I. Balslev, Phys. Rev. Lett. 76, 2492 (1996).

[15] H. Katsuragi, D. Sugino, and H. Honjo Phys. Rev. E 68, 046105 (2003).

[16] H. Katsuragi, D. Sugino, and H. Honjo, e-print cond-mat/ 0310479. 
[17] H. Katsuragi, D. Sugino, and H. Honjo, e-print cond-mat/ 0409770.

[18] X. Campi and H. Krivine, Z. Phys. A 344, 81 (1992).

[19] S. Steacy and C. Sammis, Nature (London) 353, 250 (1991).

[20] H. Inaoka and H. Takayasu, Physica A 229, 1 (1996).

[21] M. Marsili and Y. C. Zhang, Phys. Rev. Lett. 77, 3577 (1996).

[22] J. Åström and J. Timonen, Phys. Rev. Lett. 78, 3677 (1997).

[23] J. Åström, M. Kellomäki, and J. Timonen, Phys. Rev. E 55, 4757 (1997).

[24] R. Botet and M. Ploszajczak, Int. J. Mod. Phys. E 3, 1033 (1994).

[25] R. Englman, J. Phys.: Condens. Matter 3, 1019 (1991).

[26] G. Hernandez and H. J. Herrmann, Physica A 215, 420 (1995).

[27] Wm. T. Ashurst and B. L. Holian, Phys. Rev. E 59, 6742 (1999).

[28] J. A. Aström, B. L. Holian, and J. Timonen, Phys. Rev. Lett. 84, 3061 (2000).

[29] E. S. C. Ching, S. Liu, and K.-Q. Xia, Physica A 287, 83 (2000).

[30] A. Diehl, H. A. Carmona, L. E. Araripe, J. S. Andrade, Jr., and G. A. Farias, Phys. Rev. E 62, 4742 (2000).

[31] A. Bershadskii, J. Phys. A 33, 2179 (2000).

[32] A. Bershadskii and E. S. C. Ching, J. Stat. Phys. 104, 49 (2001).

[33] A. Bershadskii, Chaos, Solitons Fractals 13, 185 (2002).
[34] P. L. Krapivsky and E. Ben-Naim, Phys. Rev. E 68, 021102 (2003).

[35] F. Kun and H. J. Herrmann, Phys. Rev. E 59, 2623 (1999).

[36] B. Behera, F. Kun, S. McNamara, and H. J. Herrmann, e-print cond-mat/0404057.

[37] F. Kun and H. J. Herrmann, Comput. Methods Appl. Mech. Eng. 138, 3 (1996).

[38] F. Kun and H. J. Herrmann, Int. J. Mod. Phys. C 7, 837 (1996).

[39] F. Wittel, F. Kun, H. J. Herrmann, and B.-H. Kröplin, Phys. Rev. Lett. 93, 035504 (2004).

[40] C. Thornton, K. K. Yin, and M. J. Adams, J. Phys. D 29, 424 (1996).

[41] W. Benz and E. Asphaug, Icarus 107, 98 (1994).

[42] A. V. Potapov, M. A. Hopkins, and C. S. Campbell, Int. J. Mod. Phys. C 6, 399 (1995).

[43] S. Redner, in Statistical Models for the Fracture of Disordered Media, edited by H. J. Herrmann and S. Roux (North-Holland, Amsterdam, 1990).

[44] C. Moukarzel and H. J. Herrmann, J. Stat. Phys. 68, 911 (1992).

[45] K. B. Lauritsen, H. Puhl and H. J. Tillemans, Int. J. Mod. Phys. C 5, 909 (1994).

[46] R. Linna, Ph.D. thesis, University of Jyväskylä, Finland, 2004. 